\title{
A survey for DLA galaxies with integral field spectroscopy
}

\author{
Lise Christensen ${ }^{1}$, Sebastián. F. Sánchez ${ }^{1,2}$, Knud Jahnke ${ }^{1}$, \\ Martin M. Roth ${ }^{1}$ and Lutz Wisotzki ${ }^{1,3}$ \\ ${ }^{1}$ Astrophysikalisches Institut Potsdam, An der Sternwarte 16, 14482 Potsdam, Germany \\ email: lchristensen@aip.de \\ ${ }^{2}$ Centro Astronomico Hispano Aleman de Calar Alto, Spain \\ ${ }^{3}$ University of Potsdam, Am neuen Palais 10, 14469 Potsdam, Germany
}

\begin{abstract}
Using integral field spectroscopy (IFS) we have observed QSOs which were previously known to have intervening DLAs, to search for emission lines in the field surrounding the QSOs. The sample consists of 13 DLAs plus 7 sub-DLAs at $z>2$, from which we find emission line candidates at the expected wavelengths for 8 systems, implying a detection frequency of $40 \%$. Emission lines detected at the DLA redshifts are interpreted as being due to Ly $\alpha$ lines from galaxies associated with the DLA clouds. Candidates are detected at $3-4 \sigma$ levels in both narrow-band images and spectra created from the data cubes. We find no preference for candidate detection associated with either high or low metallicity DLAs. In comparison with the properties of previously known DLA galaxies, the candidates show similar distributions of line fluxes and line widths, but their impact parameters are slightly different, suggesting $\mathrm{HI}$ cross sections larger than $10 \mathrm{kpc}$.
\end{abstract}

\section{Introduction}

Galaxy counterparts to low-redshift damped Ly $\alpha$ absorption lines (DLAs) have indicated that DLAs are hosted by all types of gas-rich galaxies (Le Brun et al. 1997; Rao et al. 2003; Chen \& Lanzetta 2003). At low redshifts only a few DLAs are known because their confirmation needs UV spectroscopy. Despite intensive efforts, finding the galaxies responsible for DLAs at higher redshifts has proved to be difficult and time consuming with conventional techniques, and only a few $z>2$ DLA galaxies have been confirmed to date. Deep imaging campaigns to detect candidate DLA galaxies near to the QSO sight line followed by slit spectroscopy to confirm the redshifts of the candidates have provided a sample of only a handful of emission lines detected from DLA galaxies at $z \gtrsim 2$ (e.g. Warren et al. 2001; Møller et al. 2002). Other methods involve narrow-band imaging centred on Ly $\alpha$ at the DLA redshifts to look for emission from DLA galaxies (Fynbo et al. 1999). Integral field spectroscopy (IFS) provides an alternative possibility of creating adjustable narrow-band filters. Previously, two searches for emission from DLA galaxies toward two QSOs using IFS resulted in upper limits for their fluxes (Petitjean et al. 1996; Ledoux et al. 1998).

We present the first results of an IFS survey of QSOs with known intervening DLA systems using the Potsdam Multi Aperture Spectrophotometer (PMAS) mounted on the $3.5 \mathrm{~m}$ telescope at Calar Alto (Roth et al. 2005). Eight QSOs known to have a total number of 13 DLAs and 7 sub-DLAs are included in the survey. All of them have redshifts $z>2$ such that Ly $\alpha$ falls in the spectral range of the observations. Preferentially, QSOs with multiple DLAs were selected to increase the sample size. Note that this can be a potential problem because a foreground DLA galaxy can affect the emission from a background DLA galaxy due to extinction in the UV region if their lines of sight overlap. 

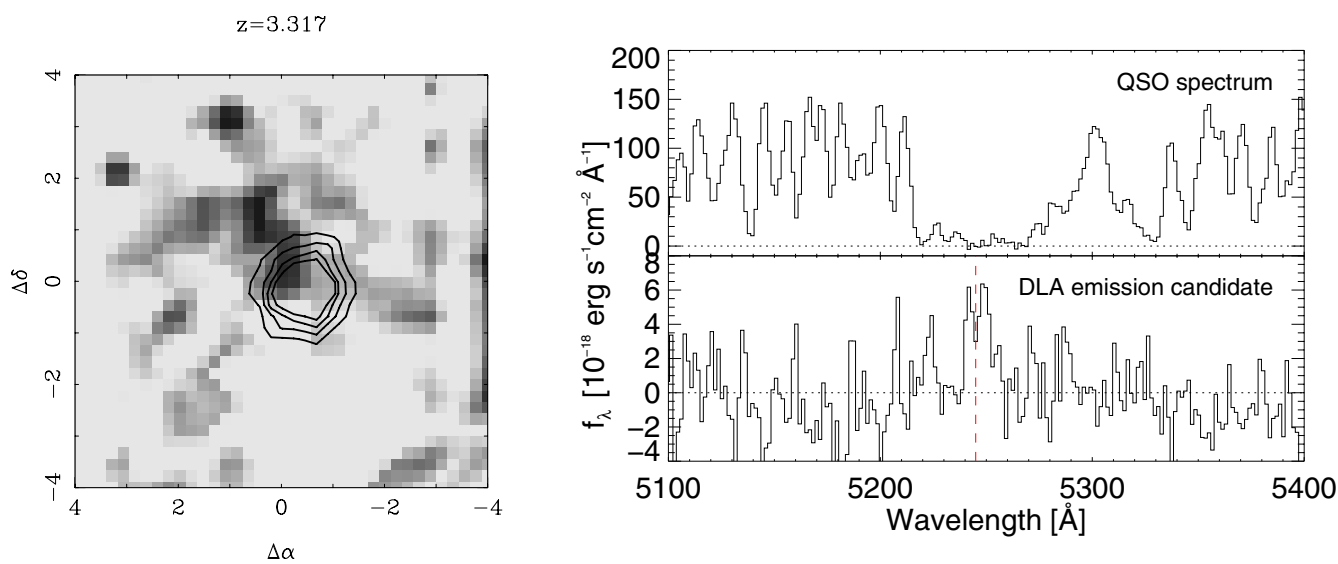

Figure 1. Candidate DLA galaxy toward PSS J2155+1358. The left hand panel shows a narrow-band image with a width of $15 \AA$ centred on Ly $\alpha$ at the DLA redshift. Contours correspond to the QSO PSF where the innermost contour shows the seeing FWHM. The right hand panel shows a section of the QSO spectrum around the DLA line in the upper panel, and the corresponding spectrum of the emission line region 1..3 to the North-East of the QSO in the narrow-band image. An emission line is present offset by $+100 \mathrm{~km} \mathrm{~s}^{-1}$ with respect to the DLA redshift which is indicated by the vertical dashed line. This line is interpreted as a candidate Ly $\alpha$ emission line from the galaxy responsible for the DLA line at $z=3.316$.

\section{Candidate detection}

The final result of PMAS data reduction for the setup used here is a data cube with $16 \times 16$ spectra covering $8^{\prime \prime} \times 8^{\prime \prime}$ on the sky, and a third spectral dimension. Inspection of data cubes was done with the Euro3D visualisation tool which allows one to interactively create narrow-band images and associated spectra (Sánchez 2004).

Narrow-band images and associated spectra were searched simultaneously for Ly $\alpha$ emission lines from the DLA galaxies. First, a narrow-band image with a typical width of $20 \AA$ was created around the redshifted Ly $\alpha$ wavelength at the DLA redshift. Several (overlapping) narrow-band images with an offset up to $\sim 15 \AA$, corresponding to $\sim \pm$ $1000 \mathrm{~km} \mathrm{~s}^{-1}$ from the DLA redshift, were inspected visually for flux over-densities. When such objects were present the corresponding spectra were selected and inspected for possible emission lines. If an emission line was present, the narrow-band filter width was adjusted to the FWHM of the emission line candidate, and a new narrow-band image was inspected for presence of other bright regions. To select good candidates we require that the candidates be detected in both the narrow-band images and spectra with a signal $3 \sigma$ above the background noise. Using this approach we found 8 good candidates out of which 2 belong to sub-DLA systems. In total 11 candidates are found, but 3 did not comply with all the criteria for being good candidates. Fig. 1 shows one of the good Ly $\alpha$ emission candidates detected in the survey.

In the case of small projected distances the candidate's Ly $\alpha$ emission line can overlap spatially with the QSO. This is the case in Fig. 1 where the candidate has an impact parameter of $\sim 1^{\prime \prime} 3$ and the seeing is $\sim 1^{\prime \prime}$. To make a cleaned spectrum of the candidate the QSO emission needs to be subtracted, which can be done in several ways. In this case, a one-dimensional spectrum in the region of the emission candidate was created from the data cube, and a scaled version of the one-dimensional QSO spectrum was subtracted. This method can also be applied in cases where the emission line candidate is offset in velocity with respect to the DLA line such that the emission line coincides 


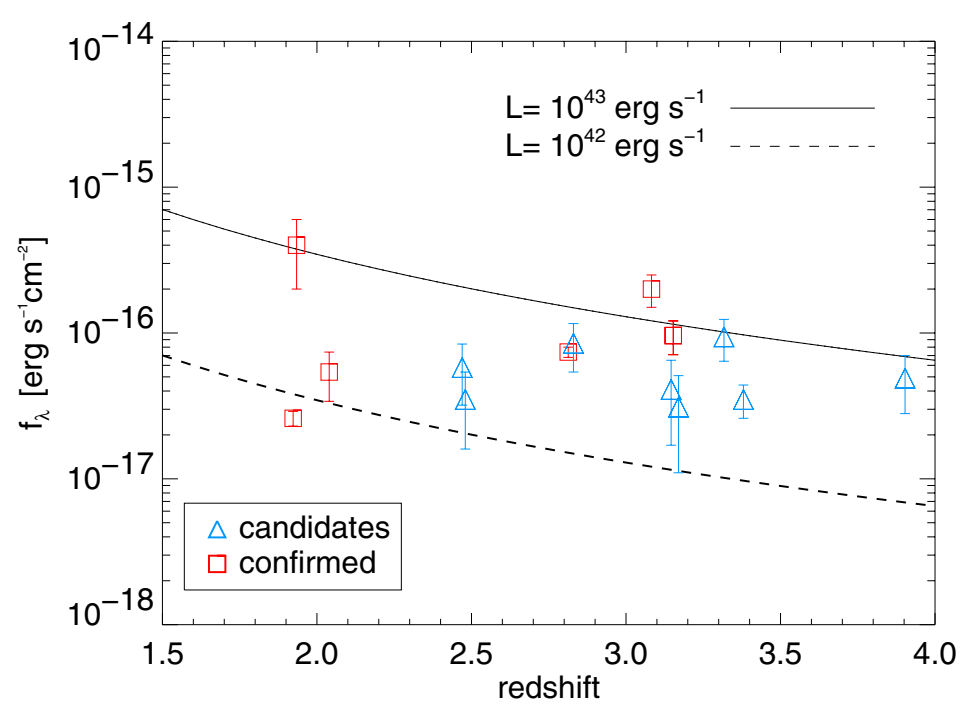

Figure 2. Total flux of the candidate Ly $\alpha$ emission lines found in this survey indicated by triangles compared to fluxes of previously known DLA galaxies (squares). Data for the known objects were taken from the literature. Total Ly $\alpha$ luminosities of $10^{43}$ and $10^{42} \mathrm{erg} \mathrm{s}^{-1}$ as a function of redshift are shown by the solid and dashed curves, respectively.

with the wings in the DLA line. However, for small impact parameters, the detection of a very faint emission line can be affected by QSO subtraction residuals.

Various other methods for subtraction of the QSO emission were tested. The smallest residuals in the spectra and hence a larger signal-to-noise ratio in the resulting onedimensional spectrum were obtained by subtracting a scaled version of the total QSO spectrum from each individual fibre spectrum before co-addition. An alternative method is to model the 3D QSO spectrum using its total spectrum and the spatial position from two-dimensional monochromatic slices in the data cube (e.g. Wisotzki et al. 2004).

\section{Results}

We strongly emphasise that our candidates are significant only at the $3-4 \sigma$ level. Bearing this in mind, we proceed with comparing their properties with those of confirmed Ly $\alpha$ emission lines from DLA galaxies described in the literature. Properties of the emission line candidates which are reported below were measured in either the co-added one-dimensional spectra or narrow-band images.

\subsection{Candidate emission lines}

In Fig. 2 we show the flux of the 8 detected candidate Ly $\alpha$ emission lines as a function of redshift. Compared to the Ly $\alpha$ fluxes of DLA galaxies that are already confirmed (see Table 1 in Weatherley et al. 2005), the candidates have line fluxes within the same range. In a flat cosmology with $\Omega_{\Lambda}=0.7$ and $H_{0}=70 \mathrm{~km} \mathrm{~s}^{-1} \mathrm{Mpc}^{-1}$, the line fluxes correspond to luminosities in the range between $10^{42}$ and $10^{43} \mathrm{erg} \mathrm{s}^{-1}$. Typical observed emission line $F W H M$ are $200-600 \mathrm{~km} \mathrm{~s}^{-1}$ which is similar to previous detected Ly $\alpha$ emission lines from DLA galaxies (Møller et al. 2002).

We also analyse the velocity offsets between the emission line candidates and the DLA redshifts reported in the literature. Seven of the candidates have offsets in the range 


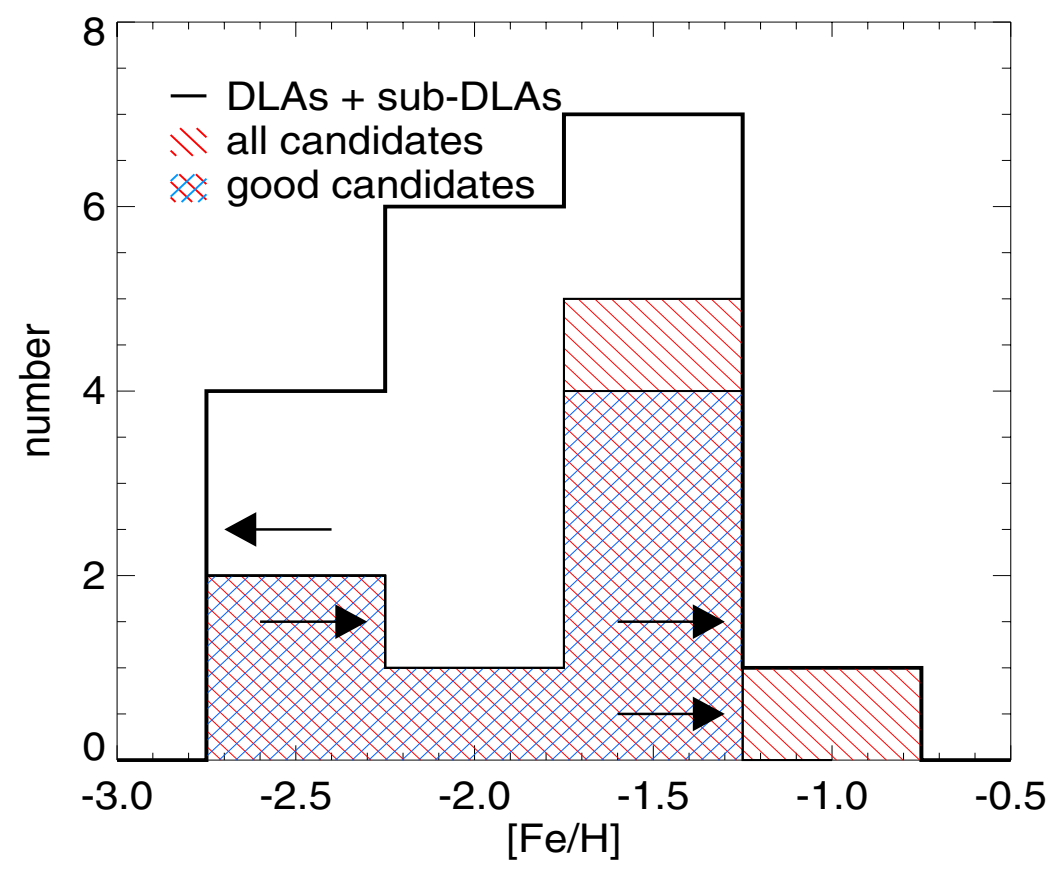

Figure 3. Metallicity distribution of all DLA and sub-DLA systems taken from the literature (solid line) compared to that of all candidates (hashed) and that of the good candidates only (cross hashed). The arrows correspond to candidates that only have limits to their metallicity determination.

0-1000 $\mathrm{km} \mathrm{s}^{-1}$, while a candidate of the DLA at $z=3.39$ toward PSS J1802 +5616 has a negative offset of $-600 \mathrm{~km} \mathrm{~s}^{-1}$. However, the uncertainties of the velocity offsets are largely due to uncertainties in the wavelength calibration, a very faint QSO spectrum that makes the detection of the DLA trough difficult, combined with possible velocity offsets between the Ly $\alpha$ absorption line and the systemic redshift of the DLA cloud. This candidate is the only example of an emission line object that can be seen immediately in the extracted spectra, i.e. before manipulating the data cube.

\subsection{Metallicities}

Space-based imaging surveys and follow up long-slit spectroscopic studies have indicated a positive metallicity-Ly $\alpha$ luminosity relation, such that Ly $\alpha$ emission is preferentially observed for higher metallicity systems (Møller et al. 2004).

We investigated the distribution of metallicities for the DLA systems with emission candidates in comparison to the total sample. Fig. 3 shows the distribution of metallicities $([\mathrm{Fe} / \mathrm{H}])$ for the DLAs with known metallicities in the survey, where metallicities have been obtained from the literature (Prochaska et al. 2003; Dessauges-Zavadsky et al. 2003, 2004). The hashed histogram shows the distribution of all the candidates irrespective of the significance, while the cross hashed histogram includes the good candidates only.

A comparison between the distribution of metallicities for the candidates and those DLA systems without candidates shows no statistically significant differences. This was found both when Fe and Si was used as metallicity tracers. Hence the question remains open until more Ly $\alpha$ emission lines are confirmed. 


\subsection{Impact parameters}

In the data cubes, impact parameters for the candidate emission lines were measured from the centroid of the objects in the narrow-band images compared to the accurate position of the QSO centroid measured in a neighbouring narrow-band image. Because of the low signal and the few bright spectra that could be identified, the spatial shapes of the emission line candidates are not regular. Experiments with very faint artificial emission lines in data cubes suggest uncertainties for the impact parameter of $\sim 0.5$.

Impact parameters for the candidates lie in the range $10-40 \mathrm{kpc}$ while two objects with very large impact parameters $(>30 \mathrm{kpc})$ lie at the edge of the field in the data cube. Potentially, these could be caused by flat-field errors which become larger at the edges in the PMAS data cubes, but they do satisfy the criteria for being good DLA candidates. The average impact parameter of $\sim 20 \mathrm{kpc}$ derived for the candidates is larger than expected by numerical simulations, favouring impact parameters of $3 \mathrm{kpc}$ for typical DLA galaxies, with only few DLA galaxies having $b>10 \mathrm{kpc}$ at both high and low redshifts (Okoshi \& Nagashima 2005; Hou et al. 2005).

Using the impact parameters for the candidates as a proxy for the sizes of neutral gas envelopes we analyse the inferred $\mathrm{H}$ I disk sizes. This is necessarily a rough approximation because it must be expected that there are large morphological differences between individual DLA galaxies.

Observations of gas disks in the local Universe have shown that they follow exponential profiles to a good approximation. Hence we fit the impact parameter distribution with an exponential function $N(\mathrm{HI})(b)=N_{0} \exp (-b / h)$, where $h$ is a scale length and $N(\mathrm{HI})$ is the measured column density of the DLA. Fig. 4 shows the resulting fit to all the candidates by the solid line, which has $\log N_{0}=20.6 \mathrm{~cm}^{-2}$ and $h=30 \pm 16 \mathrm{kpc}$. Neglecting the two candidates with the largest impact parameters gives a fit indicated by the dotted line which has $\log N_{0}=21.7 \mathrm{~cm}^{-2}$ and $h=5.1_{-1.3}^{+2.5} \mathrm{kpc}$, which is the same result obtained for a fit to the confirmed objects only. These results are consistent with DLAs arising in large disks as originally suggested by Wolfe et al. (1986).

\section{Outlook}

The objects detected with IFS in this survey need independent observations which can be done by slit-spectroscopy at the known position angle in order to confirm or reject the candidates. While this is against the main principle of using IFS, we note that the present survey is carried out at the detection limit of a 4-m class telescope. Specifically, the 8 candidate Ly $\alpha$ emission lines that are considered as good candidates are detected at only the 3-4 $\sigma$ level. If these objects are later confirmed to be real and the fluxes are in the range indicated here, data from $8 \mathrm{~m}$ class telescopes using IFS alone will allow detection of Ly $\alpha$ emission from DLA galaxies at redshifts $>2$.

We find intriguing prospects in the interpretation of impact parameters in comparison with H I profiles of local galaxies but more DLA galaxies need to be confirmed. With a larger sample of clear detections it will be feasible to estimate if high redshift DLA galaxies are indeed large spirals with impact parameters similar to those for DLA galaxies identified at lower redshifts.

\section{Acknowledgements}

L. Christensen acknowledges support by the German Verbundforschung associated with the ULTROS project, grant no. 05AE2BAA/4. 


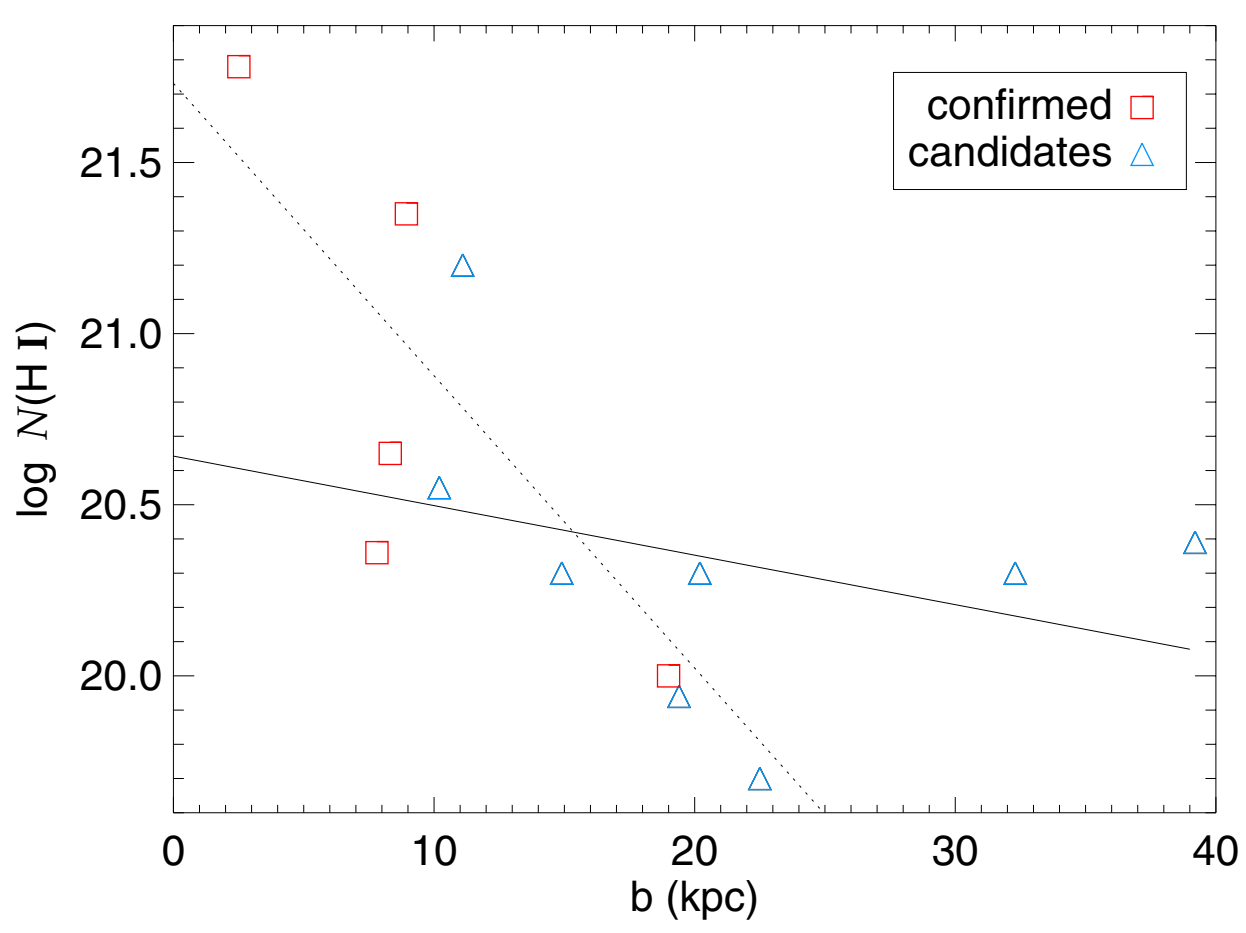

Figure 4. Column density of neutral hydrogen for the DLAs as a function of impact parameter for the candidates and previously confirmed objects. The solid line shows the fit to all the candidates and the dotted line neglects the two candidates with the largest impact parameters.

\section{References}

Chen, H.-W., Lanzetta, K. M., 2003, ApJ, 597, 706

Dessauges-Zavadsky, M., Calura, F., Prochaska, J. X., D’Odorico, S., Matteucci, F., 2004, A\&A, 416,79

Dessauges-Zavadsky, M., Péroux, C., Kim, T.-S., D’Odorico, S., McMahon, R. G., 2003, MNRAS, 345, 447

Fynbo, J. U., Møller, P., Warren, S. J., 1999, MNRAS, 305, 849

Hou, J. L., Shu, C. G., Shen, S. Y., et al., 2005, ApJ, in press, astro-ph/0501603

Le Brun, V., Bergeron, J., Boisse, P., Deharveng, J. M., 1997, A\&A, 321, 733

Ledoux, C., Theodore, B., Petitjean, P., et al., 1998, A\&A, 339, L77

Møller, P., Fynbo, J. P. U., Fall, S. M., 2004, A\&A, 422, L33

Møller, P., Warren, S. J., Fall, S. M., Fynbo, J. U., Jakobsen, P., 2002, ApJ, 574, 51

Okoshi, K., Nagashima, M., 2005, ApJ, 623, 99

Petitjean, P., Pecontal, E., Valls-Gabaud, D., Charlot, S., 1996, Nature, 380, 411

Prochaska, J., Gawiser, E., Wolfe, A., Castro, S., Djorgovski, S. G., 2003, ApJ, 595, L9

Rao, S. M., Nestor, D. B., Turnshek, D. A., et al., 2003, ApJ, 595, 94

Roth, M. M., Kelz, A., Fechner, T., et al., 2005, PASP, in press, astro-ph/0502581

Sánchez, S. F., 2004, AN, 325, 167

Warren, S. J., Møller, P., Fall, S. M., Jakobsen, P., 2001, MNRAS, 326, 759

Weatherley, S. J., Warren, S. J., Moller, P., et al., 2005, MNRAS, in press, astro-ph/0501422

Wisotzki, L., Becker, T., Christensen, L., et al., 2004, AN, 325, 135

Wolfe, A. M., Turnshek, D. A., Smith, H. E., Cohen, R. D., 1986, ApJS, 61, 249 